\title{
THE VON NEUMANN MINIMAX THEOREM REVISITED
}

\author{
HICHEM BEN-EL-MECHAIEKH \\ Department of Mathematics, Brock University \\ St. Catharines, Ontario, L2S 3A1, Canada \\ E-mail: hmechaie@brocku.ca \\ ROBERT DIMAND \\ Department of Economics, Brock University \\ St. Catharines, Ontario, L2S 3A1, Canada \\ E-mail: dimand@brocku.ca
}

1. Introduction. The aim of this note is to present a simple and elegant approach to the von Neumann Theorem in relation to contributions by Professors J. Dugundji and A. Granas [9, 10]. The M. Sion [25] generalization of the minimax theorem to quasiconcave/convex functions can be formulated as a Nonlinear Alternative [1], which turns out to be equivalent to the Dugundji-Granas version of the KKM Principle, the Browder-Ky Fan Fixed Point Theorem, and a Coincidence Principle for dual Ky Fan type set-valued maps. We include what we believe is the most elementary proof of Maurice Sion's version of the minimax theorem based on a theorem of C. Berge [4] equivalent to a Helly type result of V. Klee [15] on the intersection of a family of convex sets. This proof could easily be discussed in a first course in game theory for students whose background does not go much further than a very basic knowledge in linear algebra, advanced calculus, real analysis, and optimization.

2. From Ville to Sion: alternatives and minimax. In the May 14, 1928, session of the Académie des Sciences de Paris, Émile Borel presented a note of John von Neumann entitled Sur la théorie des jeux [18] announcing — without proof — the solution of the finite two-player, zero-sum game problem. (The note appeared a month later in the proceedings of the June 18 session.)

The problem has been posed and treated between 1921 and 1927 by Borel in a series of notes in the Comptes rendus $[5,6,7]$ as well as in his book Eléments de la théorie des

2000 Mathematics Subject Classification: Primary: 91A06, 91A13, 91A03; Secondary: 47H04, $47 \mathrm{H} 10$.

The paper is in final form and no version of it will be published elsewhere. 
probabilités [8]. Émile Borel was indeed the first to describe games in a strategic form as follows:

A two-player, zero-sum game is a triplet $(X, Y, f)$ where $X, Y$ are the sets of strategies for player 1 and player 2 respectively, and $f: X \times Y \longrightarrow \mathbb{R}$ is a so-called payoff function that player 1 attempts to maximize and player 2 tries to minimize (players are assumed to have strictly opposed interests). Each player aims at choosing security strategies, which for player 1 are of maxmin type: the number $\alpha:=\max _{X} \min _{Y} f(x, y)$ represents his guaranteed minimal gain; while for player 2 , they are of minmax type: the number $\beta:=$ $\min _{Y} \max _{X} f(x, y)$ being his guaranteed maximal loss.

It is easily seen that always, $\alpha \leq \beta$. When equality holds, the common value $\alpha=\beta$ is known as the value of the game.

In $[5,6,7]$, Émile Borel computed "by hand" minimax strategies for games with strategy sets having cardinalities 3 and 5 and announced that his constructive method would not carry to games with strategy sets having 7 or more elements. He also posed the problem as to whether or not games with arbitrary numbers of (finite) strategies always had a value. The problem he posed was precisely as follows.

Given sets of possible actions $M:=\{1, \ldots, M\}$ for player 1 , and $N:=\{1, \ldots, N\}$ for player 2. To each $i \in M, j \in N$ are associated probabilities $x_{i}, y_{j}$ respectively $\left(x_{i}, y_{j} \geq 0\right.$, $\left.\sum x_{i}=1=\sum y_{j}\right)$. The payoff function is bilinear in $x=\left(x_{i}\right)$ and $y=\left(y_{j}\right)$ :

$$
f(x, y):=\sum_{i=1}^{M} \sum_{j=1}^{N} a_{i j} x_{i} y_{j} .
$$

Does it always hold:

$$
\alpha=\max _{X} \min _{Y} f(x, y)=\min _{Y} \max _{X} f(x, y)=\beta ?
$$

Von Neumann's theorem $[18,19]$ was an affirmative answer to Borel's question. This theorem will turn out to be the starting point of the theory of strategic games as a distinct discipline. The theory soon moved on to games with $n$ players, non-constant sums of payoffs, then to infinitely many players, etc ....

Von Neumann provided a first proof based on the reduction to the cases of 2 and 3 players in Mathematische Annalen later in 1928 [19] and presented in 1932 at Menger's Colloquium in Vienna a second proof-based on Brouwer's fixed point theorem-of a closely related result on the existence of an economic equilibrium [20]. Although very elegant, both proofs constitute a challenging read, well beyond expectations for students in a first Game Theory course. John von Neumann did not publish on this topic for a decade, keeping himself busy with extensive work in functional calculus, computation theory, quantum mechanics, and with his involvement in the Manhattan Project. He was eventually persuaded by the economist Oskar Morgenstern to return his attention to game theory in Princeton during the autumn of 1940.

It is important to point out that the proof of the minimax theorem that von Neumann and Morgenstern chose to include in their celebrated book Theory of Games and Economic Behavior [AAA] was due to Jean-André Ville, a former pupil of Émile Borel $\left({ }^{1}\right)$.

$\left.{ }^{1}\right)$ It is interesting to note that not a single reference to Borel's pioneering work is made by 
Indeed, Ville edited Borel's lecture notes on psychological games Traité du calcul des probabilités et de ses applications (Paris, Gauthier-Villars, Tome IV, Fasc. 2, 1938). In an appendix to the Traité a note appears entitled Sur la théorie générale des jeux où intervient l'habileté des joueurs where Ville provides a remarkably simple proof of von Neumann's minimax theorem as well as a generalization to continuous strategy sets, and for the first time, outlines the importance of convexity in the existence of minimax solutions (see Theorem 1 below). This connection to convex sets will subsequently be exploited by many authors (S. Kakutani, J. Nash, etc ... ) as to become fundamental in the evolution of the theory into a branch of modern mathematics.

A number of generalizations of von Neumann's theorem were published in the years following the end of World War II by M. Shiffman [24] (extension to concave-convex function, 1949), H. Weyl [27] (1950), H. Kneser [17] (extension to concave-convex semi-continuous functions, 1952), Ky Fan [11] (concave-convex semi-continuous functions, 1953), and C. Berge [3] (concave-convex semi-continuous functions, 1954). In 1954 H. Nikaidô [23] used the Brouwer fixed point theorem to extend the result to quasiconcave-convex continuous functions, and in 1958, M. Sion [25] explicitly, and for the first time, used the Knaster-Kuratowski-Mazurkiewicz (KKM) theorem [16] to derive a minimax for quasiconcave-convex and upper-lower- semi-continuous. Our focus in what follows shall be on this extension of the von Neumann theorem.

Let us start by pointing out that the key result of Ville's paper (Theorem in [26]) (from which the von Neumann theorem readily derives) can be stated as a linear alternative:

TheOREM 1 (Ville's Linear Alternative). Let $\mathbb{R}_{+}^{n}$ be the non-negative cone in the Euclidean space $\mathbb{R}^{n}$, let $\Delta$ be the standard simplex in $\mathbb{R}^{p}$, and let $\left\{f_{1}, \ldots, f_{p}\right\}$ be any given collection of linear forms on $\mathbb{R}^{n}$. Then the following alternative holds: or

A) there exists $\bar{x} \in \mathbb{R}_{+}^{n}$ such that $\sum_{j=1}^{p} y_{j} f_{j}(\bar{x})<0$, for every $y=\left(y_{1}, \ldots, y_{p}\right) \in \Delta$;

B) there exists $\bar{y}=\left(\bar{y}_{1}, \ldots, \bar{y}_{p}\right) \in \Delta$ such that $\sum_{j=1}^{p} \bar{y}_{j} f_{j}(x) \geq 0$ for every $x \in \mathbb{R}_{+}^{n}$.

Much in the same spirit, Maurice Sion's generalization of the minimax theorem-a significant extension to nonlinear payoff functions - derives from a nonlinear alternative for quasiconvex-concave functions.

Definition 2. A real function $f: X \longrightarrow \mathbb{R}$ defined on a subset $X$ of a topological vector space is:

(i) quasiconvex if for each $\lambda \in \mathbb{R}$, the level set $\{x \in X: f(x) \leq \lambda\}$ is a convex subset of $X$;

(ii) lower semicontinuous (l.s.c.) if for each $\lambda \in \mathbb{R}$, the level set $\{x \in X: f(x) \leq \lambda\}$ is a closed subset of $X$.

$f$ is quasiconcave if $-f$ is quasiconvex; it is upper semicontinuous (u.s.c.) if $-f$ is l.s.c. Note that $f$ is quasiconvex on $X$ if and only if $f\left(\mu x_{1}+(1-\mu) x_{2}\right) \leq \max \left\{f\left(x_{1}\right), f\left(x_{2}\right)\right\}$

von Neumann and Morgenstern; an early sign of a controversy that would become public in 1953 with the English translation in the journal Econometrica of the three early papers of Borel together with an introduction and commentaries by Maurice Fréchet [12] and a communication by John von Neumann [21] (see Ben-El-Mechaiekh and Dimand [2]). 
for all $x_{1}, x_{2} \in X$ and all $\mu \in[0,1]$; and that the upper envelope $\sup \left\{f_{i}: i \in I\right\}$ of a family of quasiconvex (respectively, l.s.c.) functions is quasiconvex (l.s.c., respectively).

The key observation leading to the Sion-von Neumann theorem is the following alternative:

Theorem 3 (A Nonlinear Alternative [1]). Let $X$ and $Y$ be two convex subsets of topological vector spaces and let $\tilde{f}, f, g, \tilde{g}: X \times Y \longrightarrow \mathbb{R}$ be four functions satisfying:

(i) $\tilde{f}(x, y) \leq f(x, y) \leq g(x, y) \leq \tilde{g}(x, y)$, for all $(x, y) \in X \times Y$;

(ii) $y \mapsto \tilde{f}(x, y)$ is lower semicontinuous on $Y$, for each fixed $x \in X$;

(iii) $x \mapsto f(x, y)$ is quasiconcave on $X$, for each fixed $y \in Y$;

(iv) $y \mapsto g(x, y)$ is quasiconvex on $Y$, for each fixed $x \in X$;

(v) $x \mapsto \tilde{g}(x, y)$ is upper semicontinuous on $X$, for each fixed $x \in X$.

If $Y$ is compact, then for any $\lambda \in \mathbb{R}$, the following alternative holds:

A) there exists $\bar{x} \in X$ such that $\tilde{g}(\bar{x}, y) \geq \lambda$, for all $y \in Y$ or

B) there exists $\bar{y} \in Y$ such that $\tilde{f}(x, \bar{y}) \leq \lambda$, for all $x \in X$.

In case $\lambda=0$ and $X=\mathbb{R}_{+}^{n}$ and $Y=\Delta$, clearly a compact set, we obtain a slightly weaker version of Ville's linear alternative with $f(x, y)=\tilde{f}(x, y)=g(x, y)=\tilde{g}(x, y)=$ $-\sum_{j=1}^{p} y_{j} f_{j}(x)$ a bilinear form in the variables $\left(x, y=\left(y_{j}\right)\right) \in \mathbb{R}_{+}^{n} \times \Delta$.

Corollary 4. The Nonlinear Alternative above is equivalent to

$$
\tilde{\alpha}=\sup _{X} \inf _{Y} \tilde{g}(x, y) \geq \inf _{Y} \sup _{X} \tilde{f}(x, y)=\tilde{\beta} .
$$

Proof. One readily verifies that if $\tilde{\alpha} \geq \tilde{\beta}$ and thesis (A) in Theorem 3 fails, then $\exists \bar{y} \in Y$ such that $\sup _{X} \tilde{f}(x, \bar{y}) \leq \lambda$ and $(\mathrm{B})$ is thus satisfied. Conversely, assume that $\tilde{\alpha}<\tilde{\beta}<\infty$. Let $\lambda$ be an arbitrary but fixed real number strictly between $\tilde{\alpha}$ and $\tilde{\beta}$. By the Nonlinear Alternative, either there exists $\bar{y} \in Y$ such that $\tilde{f}(x, \bar{y}) \leq \lambda$, for all $x \in X$ thus $\tilde{\beta} \leq \lambda<\tilde{\beta}$ which is impossible, or there exists $\bar{x} \in X$ such that $\tilde{g}(\bar{x}, y) \geq \lambda$, for all $y \in Y$ thus $\tilde{\alpha} \geq \lambda>\tilde{\alpha}$ which is absurd. Hence $\tilde{\alpha} \geq \tilde{\beta}$.

Maurice Sion's formulation of the minimax theorem follows immediately with $\tilde{f}=$ $f=g=\tilde{g}$ :

TheOREm 5 (Theorem 3.4 in [25]). Let $X$ and $Y$ be convex subsets of topological vector spaces, with $Y$ compact, and let $f$ be a real function on $X \times Y$ such that:

(i) $x \mapsto f(x, y)$ is quasiconcave and upper semicontinuous on $X$, for each fixed $y \in Y$;

(ii) $y \mapsto f(x, y)$ is quasiconvex and lower semicontinuous on $Y$, for each fixed $x \in X$.

Then (1) holds, i.e.,

$$
\alpha=\max _{X} \min _{Y} f(x, y)=\min _{Y} \max _{X} f(x, y)=\beta .
$$

Proof. If $\tilde{f}=f=g=\tilde{g}$, then $\tilde{\alpha}=\alpha:=\inf _{Y} \sup _{X} f(x, y), \tilde{\beta}=\beta:=\sup _{X} \inf _{Y} f(x, y)$. Since the inequality $\alpha \leq \beta$ is always true, it follows that $\alpha=\beta$.

A simple proof of the Sion theorem was recently provided by J. Kindler [14]. We end this section with a simpler and very elementary proof based on the following result of Claude Berge. 
Lemma 6 (Berge [4]). Let $C$ and $C_{1}, \ldots, C_{n}$ be closed convex sets in a Euclidean space satisfying:

(i) $C \cap \bigcap_{i=1, i \neq j}^{n} C_{i} \neq \emptyset$ for any $j=1,2, \ldots, n$;

(ii) $C \cap \bigcap_{i=1}^{n} C_{i}=\emptyset$.

Then $C \nsubseteq \bigcup_{i=1}^{n} C_{i}$.

Proof of the Nonlinear Alternative when $\tilde{f}=f=g=\tilde{g}$. For simplicity, we limit ourselves to the setting of the Sion theorem with $\tilde{f}=f=g=\tilde{g}$. Assuming that the Nonlinear Alternative does not hold, i.e., both (A) and (B) fail, amounts to assuming that the sets $X$ and $Y$ can be covered by the collections of open level sets $\left\{U_{y}:=\{x \in X\right.$ : $f(x, y)<\lambda\}: y \in Y\}$ and $\left\{O_{x}:=\{y \in Y: f(x, y)>\lambda\}: x \in X\right\}$ respectively. $Y$ being compact, it can be covered by a finite subcollection $\left\{O_{x_{k}}: k=1, \ldots, m\right\}$. The convex hull $C:=\operatorname{Conv}\left\{x_{k}: k=1, \ldots, m\right\}$ lies in a finite-dimensional subspace $L$ of the linear topological space containing $X$ and is compact; thus, it can be covered by a finite subcollection $\left\{U_{y_{i}}: i=1, \ldots, n\right\}$. One can drop elements of $\left\{y_{i}: i=1, \ldots, n\right\}$ as to make the cover $\left\{U_{y_{i}}\right\}$ minimal, in the sense that $C \subseteq \bigcup_{i=1}^{n} U_{y_{i}}$ but $C \nsubseteq \bigcup_{i=1, i \neq j}^{n} U_{y_{i}}$ for any $j=1, \ldots, n$. For any $i=1,2, \ldots, n$, let $C_{i}:=\left\{x \in L: f\left(x, y_{i}\right) \geq \lambda\right\}$, a convex and compact subset of $L$. The fact that $C$ is covered by $\left\{U_{y_{i}}\right\}$ is precisely the emptiness of the intersection $C \cap \bigcap_{i=1}^{n} C_{i}$. The minimality of $\left\{U_{y_{i}}\right\}$ is nothing else than $C \cap \bigcap_{i=1, i \neq j}^{n} C_{i} \neq \emptyset$ for any $j=1,2, \ldots, n$. By Lemma 6 , there exists $x_{0} \in C$ with $f\left(x_{0}, y_{i}\right)<\lambda$ for all $i=1,2, \ldots, n$. The quasiconvexity of $f\left(x_{0},.\right)$ implies that

$\exists x_{0} \in C$ such that $f\left(x_{0}, y\right)<\lambda$ for all $y \in D:=\operatorname{Conv}\left\{y_{i}: i=1, \ldots, n\right\}$.

A similar argument yields

$$
\exists y_{0} \in D \text { such that } f\left(x, y_{0}\right)>\lambda \text { for all } x \in C .
$$

Thus,

$$
\lambda<f\left(x_{0}, y_{0}\right)<\lambda
$$

a contradiction.

Lemma 6 is equivalent to the Preliminary result in V. L. Klee Jr. [15]. For the sake of completeness, we reproduce Klee's simple proof based on the separation of convex compact sets by a hyperplane in Euclidean spaces (this separation property is commonly taught at the beginning of a first course on continuous optimization).

Proof of Lemma 6. One may assume with no loss of generality that $C$ and the $C_{i}$ 's are compact (otherwise, replace $C$ by the convex finite polytope $C^{\prime}:=\operatorname{Conv}\left\{y_{j}\right.$ : $j=1, \ldots, n\}, y_{j} \in C \cap \bigcap_{i=1, i \neq j}^{n} C_{i}$, and $C_{i}$ by $\left.C_{i}^{\prime}:=C_{i} \cap C^{\prime}\right)$. The proof is by induction on $n$. If $n=1$, (i) asserts that $C$ is non-empty and (ii) that $C$ and $C_{1}$ are disjoint. Thus, clearly $C \nsubseteq C_{1}$. 
Suppose Lemma 4 holds for $n=k-1$ and consider the case $n=k$, i.e., $C,\left\{C_{i}\right\}_{i=1}^{k}$ is a collection of compact convex sets such that $C \cap \bigcap_{i=1, i \neq j}^{k} C_{i} \neq \emptyset$, for all $j=1, \ldots, k$ and $\left(C \cap C_{k}\right) \cap \bigcap_{i=1}^{k-1} C_{i}=\emptyset$. The disjoint compact convex sets $\left(C \cap C_{k}\right)$ and $\bigcap_{i=1}^{k-1} C_{i}$ can be strictly separated by a hyperplane $H$. Putting $C^{\prime}:=H \cap C$ and $C_{i}^{\prime}:=H \cap C_{i}$, it follows at once that $C^{\prime} \cap \bigcap_{i=1}^{k-1} C_{i}^{\prime}=\emptyset$. Moreover, for a given arbitrary $j_{0} \in\{1, \ldots, k-1\}$, let $y_{0} \in C \cap \bigcap_{i=1, i \neq j_{0}}^{k} C_{i}$, thus $y_{0} \in C \cap C_{k}$, and let $y_{k} \in C \cap \bigcap_{i=1}^{k-1} C_{i}$ be arbitrary. Clearly, the points $y_{0}$ and $y_{k}$ are strictly separated by $H$. The intersection $\bar{z}$ of the line segment $\left[y_{0}, y_{k}\right]$ with $H$ belongs to $C$ as well as to $\bigcap_{i=1, i \neq j_{0}}^{k-1} C_{i}$. $j_{0}$ being arbitrary, hypotheses (i) and (ii) are verified for the collection $C^{\prime},\left\{C_{i}^{\prime}\right\}_{i=1}^{k-1}$. By induction hypothesis, $C^{\prime}=H \cap C \nsubseteq \bigcup_{i=1}^{k-1} C_{i}=\bigcup_{i=1}^{k-1} C_{i} \cap H$. Since $(H \cap C) \cap C_{k}=\emptyset$, it follows that $H \cap C \nsubseteq \bigcup_{i=1}^{k} C_{i} \cap H$, thus $C \nsubseteq \bigcup_{i=1}^{k} C_{i}$.

\section{Nonlinear alternative, KKM, coincidence and fixed points: a circular tour.} We proceed now to show that the Nonlinear Alternative (in its generality) is equivalent to the KKM theorem and to a coincidence theorem of Ky Fan type. But first we recall the basic ingredients and key results.

Definition 7. Given an arbitrary subset $X$ of a real vector space $E$, a set-valued map $\Gamma: X \longrightarrow 2^{E}$ is said to be $K K M$ if for every finite subset $A:=\left\{x_{1}, \ldots, x_{n}\right\} \subseteq X$

$$
\operatorname{Conv}(A) \subset \bigcup_{i=1}^{n} \Gamma\left(x_{i}\right) .
$$

Theorem 8 (Dugundji-Granas KKM Theorem $[9,10,13]$ ). Let $\Gamma, \tilde{\Gamma}: X \longrightarrow 2^{E}$ be two set-valued maps defined on an arbitrary subset $X$ of a real topological vector space $E$ and verifying:

(i) $\Gamma$ is a selection of $\tilde{\Gamma}$ (i.e., $\Gamma(x) \subseteq \tilde{\Gamma}(x)$ for all $x \in X)$;

(ii) $\Gamma$ is a KKM map;

(iii) all values of $\tilde{\Gamma}$ are closed and at least one is compact;

(iv) $\bigcap_{x \in X} \tilde{\Gamma}(x) \neq \emptyset \Rightarrow \bigcap_{x \in X} \Gamma(x) \neq \emptyset$.

Then $\bigcap_{x \in X} \Gamma(x) \neq \emptyset$.

Given two non-empty subsets $X$ and $Y$ in topological vector spaces, let us say that: Definition 9 ([1]). A set valued map $A: X \longrightarrow 2^{Y}$ is a $\Phi$-map (written $A \in \Phi(X, Y)$ ) if and only if:

(i) $A^{-1}(y)$ is convex in $X$ for all $y \in Y$;

(ii) $A$ has a multivalued selection with open values and non-empty fibers, i.e., $A(x) \supseteq$ $\tilde{A}(x)$ open in $Y$ for all $x \in X$, and $\tilde{A}^{-1}(y) \neq \emptyset$ for all $y \in Y$. 
A map $B: X \longrightarrow 2^{Y}$ is a $\Phi^{*}-m a p$ if and only if its inverse $B^{-1}: Y \longrightarrow 2^{X}$ is a $\Phi$-map.

Theorem 10 (A Coincidence Principle [1]). Let $A \in \Phi(X, Y)$ and $B \in \Phi^{*}(X, Y)$ where $X$ and $Y$ are non-empty convex subsets of topological vector spaces. If $Y$ is compact, then there exists $\left(x_{0}, y_{0}\right) \in X \times Y$ such that

$$
y_{0} \in A\left(x_{0}\right) \cap B\left(x_{0}\right) .
$$

This coincidence principle can be seen as a fixed point theorem for the composition of the two $\Phi$-maps $B^{-1} \circ A: X \longrightarrow 2^{X}$ through the compact convex set $Y$. This fixed point principle also holds for one $\Phi$-map:

Theorem 11 (The Browder-Ky Fan Fixed Point Theorem [1]). If $A \in \Phi^{*}(X, Y)$ (equivalently $A \in \Phi(X, Y)$ ) where $X$ is a convex compact subset of a topological vector space, then $A$ has a fixed point $x_{0} \in A\left(x_{0}\right)$.

We are ready to formulate the main theorem of this paper.

Theorem 12 (A Circular Tour). The Nonlinear Alternative $\stackrel{1}{\Rightarrow}$ The Coincidence Principle $\stackrel{2}{\Rightarrow}$ The Browder-Ky Fan Fixed Point Theorem $\stackrel{3}{\Rightarrow}$ The Brouwer Fixed Point Theorem $\stackrel{4}{\Rightarrow}$ The Dugundji-Granas KKM Theorem $\stackrel{5}{\Rightarrow}$ The Nonlinear Alternative.

Proof. (1) The Nonlinear Alternative $\Rightarrow$ The Coincidence Principle.

Assume that the hypotheses of the Coincidence Principle hold. Define the functions $\tilde{f}, f, g, \tilde{g}: X \times Y \longrightarrow \mathbb{R}$ as:

$$
\begin{aligned}
& \tilde{f}(x, y):=\left\{\begin{array}{ll}
0 & \text { if } y \notin \tilde{A}(x) \\
1 & \text { if } y \in \tilde{A}(x)
\end{array} \quad f(x, y):= \begin{cases}0 & \text { if } y \notin A(x) \\
1 & \text { if } y \in A(x)\end{cases} \right. \\
& g(x, y):=\left\{\begin{array}{ll}
0 & \text { if } y \in B(x) \\
1 & \text { if } y \notin B(x)
\end{array} \quad \tilde{g}(x, y):= \begin{cases}0 & \text { if } y \in \tilde{B}(x) \\
1 & \text { if } y \notin \tilde{B}(x) .\end{cases} \right.
\end{aligned}
$$

It is obvious that for all $(x, y) \in X \times Y$, we have $\tilde{f}(x, y) \leq f(x, y)$ and that $g(x, y) \leq$ $\tilde{g}(x, y)$ and that hypotheses (ii) to (v) of the Nonlinear Alternative hold. The assumptions $\tilde{B}(x) \neq \emptyset$ for all $x \in X$ and $\tilde{A}^{-1}(y) \neq \emptyset$ for all $y \in Y$ are equivalent to the failure of conclusions (A) and (B) of the Nonlinear Alternative. Necessarily hypotheses (i) of the alternative must fail! More precisely, the inequality

$$
f(x, y) \leq g(x, y) \text { for all }(x, y) \in X \times Y
$$

fails.

That is:

$$
0=g\left(x_{0}, y_{0}\right)<\frac{1}{2}<f\left(x_{0}, y_{0}\right)=1 \text { for some }\left(x_{0}, y_{0}\right) \in X \times Y,
$$

which is equivalent to $y_{0} \in A\left(x_{0}\right) \cap B\left(x_{0}\right)$.

(2) The Coincidence Principle $\Rightarrow$ The Browder-Ky Fan Fixed Point Theorem.

The proof of this implication is, as far as we can tell, new (but more convoluted than that of the converse); it proceeds in three steps. 
The first step is to reduce the Browder-Ky Fan fixed point theorem to finite dimension by using a standard selection argument (see [1]).

Indeed, given a map $A \in \Phi^{*}(X, Y)$, where $X$ is compact and $Y$ is convex, each in a topological vector space, the fact that $A(x) \neq \emptyset$ for all $x \in X$ implies that the collection $\mathcal{O}:=\left\{A^{-1}(y): y \in Y\right\}$ of open subsets of $X$ forms a cover of $X$. By compactness, $X$ can be covered by a finite subcollection $\mathcal{O}_{f}:=\left\{A^{-1}\left(y_{i}\right): i=1, \ldots, n\right\}$. Let $\left\{\lambda_{i}: X \longrightarrow[0,1]\right\}_{i=1}^{n}$ be a continuous partition of unity subordinated to the finite cover $\mathcal{O}_{f}$ and define a continuous single-valued mapping $s: X \longrightarrow Y$ by

$$
s(x):=\sum_{i=1}^{n} \lambda_{i}(x) y_{i}, \text { a convex combination } \forall x \in X .
$$

Since, for any given $x \in X, \lambda_{i}(x) \neq 0 \Rightarrow x \in A^{-1}\left(y_{i}\right) \Leftrightarrow y_{i} \in A(x) \Rightarrow s(x) \in A(x)$ because $A(x)$ is convex, the mapping $s$ is:

- a continuous selection of $A$, and

- finite-dimensional, because $s(X) \subseteq C=\operatorname{Conv}\left\{y_{1}, \ldots, y_{n}\right\}$ a convex finite-dimensional polytope $\subseteq Y$.

Now if $X=Y$, the restriction/compression map $A_{f}:=\left.A\right|_{C} \cap C: C \longrightarrow 2^{C}$ defined by

$$
A_{f}(x)=A(x) \cap C \quad \forall x \in C
$$

is certainly a $\Phi^{*}$-map $\left(\forall x, y \in C, A_{f}^{-1}(y)\right.$ is open in $X$, thus open in $C$, moreover $s(x) \in$ $A_{f}(x) \neq \emptyset$ and $A_{f}(x)$ is convex). It is clear that a fixed point for $A_{f}$ is also a fixed point for $A$. The Browder-Ky Fan fixed point theorem has thus been reduced to convex finite-dimensional polytopes.

The second step consists in showing that in finite dimension, the Coincidence Principle implies a coincidence result between a single-valued mapping and a $\Phi$-map, more precisely:

Proposition 13. Let $C$ be a convex compact subset in a Euclidean space $\mathbb{R}^{m}$, let $f$ : $C \longrightarrow C$ be a single-valued continuous mapping, and let $A \in \Phi(C)$. Then, there exists $x_{0} \in C$ with

$$
f\left(x_{0}\right) \in A\left(x_{0}\right) .
$$

The proof of this Proposition requires

Lemma 14. Given a continuous function $f: C \longrightarrow Y$ from a compact metric space $C$ into a convex subset $Y$ of a normed space, for any $\epsilon>0$, there exists a $\Phi^{*}$-map $\Phi_{\epsilon}: C \longrightarrow 2^{Y}$ such that

$$
f(x) \in \Phi_{\epsilon}(x) \subset B_{\epsilon}\left(f\left(B_{\epsilon}(x)\right)\right) \quad \forall x \in C,
$$

where, for a given set $Z, B_{\epsilon}(Z)$ denotes the $\epsilon$-open ball around $Z$.

Proof. This lemma says that every continuous function $f$ on a compact set admits a $\Phi^{*}$-majorant that is within an $\epsilon$-tubular neighborhood of the graph of $f$. It is a converse to the selection property for $\Phi^{*}$-maps discussed above. Its proof is as follows.

By continuity, for all $x \in C$ there exists $\delta_{x} \in(0, \epsilon)$ with $f\left(B_{\delta_{x}}(x) \cap C\right) \subset B_{\epsilon}(f(x)) \cap Y$. Let $\left\{B_{\delta_{x_{i}}}\left(x_{i}\right) \cap C: i=1, \ldots, p\right\}$ be a finite cover of the compact set $C$, and for each 
$x \in C$, let $I(x):=\left\{i \in\{1, \ldots, p\}: x \in B_{\delta_{x_{i}}}\left(x_{i}\right)\right\}$ be the set of essential indices of $x$ w.r.t. the open cover. Define the map $\Phi_{\epsilon}$ by setting

$$
\Phi_{\epsilon}(x):=\bigcap_{i \in I(x)}\left(B_{\epsilon}\left(f\left(x_{i}\right)\right) \cap Y\right) \quad \forall x \in C .
$$

Clearly, the values of $\Phi_{\epsilon}$ are convex non-empty and its graph is open (indeed, for each $x \in C$, the open set $\bigcap_{i \in I(x)}\left(B_{\delta_{x_{i}}}\left(x_{i}\right) \cap C\right) \times \Phi_{\epsilon}(x)$ is a neighborhood of $\left.\{x\} \times \Phi_{\epsilon}(x)\right)$, thus $\Phi_{\epsilon}$ has open fibers, thus $\Phi_{\epsilon} \in \Phi^{*}(C, Y)$. By definition, $f(x) \in \Phi_{\epsilon}(x) \subset B_{\epsilon}\left(f\left(B_{\epsilon}(x)\right)\right)$ for all $x \in C$.

Proof of Proposition 13. Let $f: C \longrightarrow C$ be continuous, $A \in \Phi(C)$, and let $s: C \longrightarrow C$ be a continuous selection of the map $A^{-1} \in \Phi^{*}(C)$ and, for a fixed but arbitrary $\epsilon>0$, let $\Phi_{\epsilon}, \Psi_{\epsilon}: C \longrightarrow 2^{C}$ be the two $\Phi^{*}$ enlargements of $f$ and $s$ provided by Lemma 14, i.e.

$$
\begin{gathered}
f(x) \in \Phi_{\epsilon}(x) \subset B_{\epsilon}\left(f\left(B_{\epsilon}(x)\right)\right) \quad \forall x \in C, \\
s(y) \in \Psi_{\epsilon}(y) \subset B_{\epsilon}\left(s\left(B_{\epsilon}(y)\right)\right) \quad \forall y \in C .
\end{gathered}
$$

By the Coincidence Principle, there exists $\left(x_{\epsilon}, y_{\epsilon}\right) \in C \times C$ with

$$
y_{\epsilon} \in \Phi_{\epsilon}\left(x_{\epsilon}\right) \cap \Psi_{\epsilon}^{-1}\left(x_{\epsilon}\right) .
$$

By compactness, as $\epsilon \rightarrow 0$, a subnet of $\left(x_{\epsilon}, y_{\epsilon}\right)$ converges to some $\left(x_{0}, y_{0}\right)$ in $C \times C$. Since $f$ and $s$ are continuous, inclusions (3) imply that $x_{0}=s\left(y_{0}\right) \in A^{-1}\left(y_{0}\right)$ and $y_{0}=f\left(x_{0}\right)$.

The last step, in order to establish the Browder-Ky Fan fixed point theorem in finite dimension, simply consists in taking, in Proposition 13, $f=\operatorname{Id}_{C}$, the identity mapping.

(3) The Browder-Ky Fan Fixed Point Theorem $\Rightarrow$ The Brouwer Fixed Point Theorem.

Let $C$ be a non-empty convex compact subset of $\mathbb{R}^{n}$ and let $f: C \longrightarrow C$ be a continuous mapping. Let $\varphi(x, y):=\langle y-f(y), y-x\rangle$ and define a map $A: C \longrightarrow 2^{C}$ by

$$
A(x):=\{y \in C: \varphi(x, y)>0\}, \quad x \in C .
$$

The fibers $A^{-1}(y)$ of $A$ are obviously convex due to the linearity of $x \mapsto \varphi(x, y)$, and the images $A(x)$ of $A$ are open due to the continuity of $y \mapsto \varphi(x, y)$. Assume that $A$ is surjective. Then it is a $\Phi$-map with $\tilde{A}=A$, and must have a fixed point $x_{0} \in A\left(x_{0}\right)$ by the Browder-Ky Fan fixed point theorem. But this is impossible as $\varphi\left(x_{0}, x_{0}\right)=0$. Hence, $A$ is not surjective:

$$
\exists \bar{y} \in C \text { with } A^{-1}(\bar{y})=\emptyset \text {, i.e. }\langle\bar{y}-f(\bar{y}), \bar{y}-x\rangle \leq 0 \quad x \in C,
$$

in particular for $x=f(\bar{y})$. This ends the proof as $0 \leq\|\bar{y}-f(\bar{y})\|^{2} \leq 0$, i.e. $\bar{y}=f(\bar{y})$.

(4) The Brouwer Fixed Point Theorem $\Rightarrow$ The Dugundji-Granas KKM Theorem.

Observe first that since all values of $\tilde{\Gamma}$ are closed and that at least one say $\tilde{\Gamma}(\bar{x})$-is compact, then the inclusion $\bigcap_{x \in X} \tilde{\Gamma}(x) \subseteq \tilde{\Gamma}(\bar{x})$ implies that it suffices for the conclusion of Theorem 8 to hold, that the family $\{\tilde{\Gamma}(x): x \in X\}$ has the finite intersection property, that is $\bigcap_{i=1}^{n} \tilde{\Gamma}\left(x_{i}\right) \neq \emptyset$ for any finite subset $\left\{x_{1}, \ldots, x_{n}\right\}$ of $X$. Assume for a contradiction 
that there exists a finite subset $\left\{x_{1}, \ldots, x_{n}\right\}$ of $X$ such that $\bigcap_{i=1}^{n} \tilde{\Gamma}\left(x_{i}\right)=\emptyset$. Let $C:=$ $\operatorname{Conv}\left\{x_{1}, \ldots, x_{n}\right\}$ and, for each $i=1, \ldots, n$, consider the closed set $F_{i}=C \cap \tilde{\Gamma}\left(x_{i}\right)$. If $d$ is the Euclidean metric on the finite-dimensional space spanned by $\left\{x_{1}, \ldots, x_{n}\right\}$, then $d\left(x, F_{i}\right)=0 \Leftrightarrow x \in F_{i}$. Since $\bigcap_{i=1}^{n} F_{i}=\emptyset$, it follows that the function $\mu: C \longrightarrow \mathbb{R}$ defined by $\mu(x):=\sum_{i=1}^{n} d\left(x, F_{i}\right)$ is positive for each $x \in C$. Define a continuous mapping $f: C \longrightarrow C$ by setting

$$
f(x):=\frac{1}{\mu(x)} \sum d\left(x, F_{i}\right) x_{i}, \quad x \in C .
$$

By the Brouwer fixed point theorem, $f$ has in $C=\operatorname{Conv}\left\{x_{1}, \ldots, x_{n}\right\}$ a fixed point $x_{0}=f\left(x_{0}\right)$. Since $\Gamma$ is KKM, it follows that $x_{0} \in \bigcup_{i=1}^{n} \Gamma\left(x_{i}\right) \subseteq \bigcup_{i=1}^{n} \tilde{\Gamma}\left(x_{i}\right)$.

(5) The Dugundji-Granas KKM Theorem $\Rightarrow$ The Nonlinear Alternative.

Step 1. For simplicity, consider first the case of the nonlinear alternative with both $X$ and $Y$ convex compact. Define set-valued maps $F, \tilde{F}: X \longrightarrow 2^{Y}$ and $G, \tilde{G}: Y \longrightarrow 2^{X}$ by:

$$
\begin{array}{ll}
F(x):=\{y \in Y: f(x, y)>\lambda\}, & \tilde{F}(x):=\{y \in Y: \tilde{f}(x, y)>\lambda\}, \\
G(y):=\{x \in X: g(x, y)<\lambda\}, & \tilde{G}(y):=\{x \in X: \tilde{g}(x, y)<\lambda\} .
\end{array}
$$

Clearly, $\tilde{F}(x) \subseteq F(x)$ for all $x \in X$, and $\tilde{G}(y) \subseteq G(y)$ for all $y \in Y$.

Case 1. $\tilde{F}$ is not surjective or $\tilde{G}$ is not surjective.

Case 2. Both $\tilde{F}$ and $\tilde{G}$ are surjective.

In Case 1 the Nonlinear Alternative holds (indeed, conclusion (B) of the alternative corresponds to " $\tilde{F}$ is not surjective", whereas the statement " $\tilde{G}$ is not surjective" is precisely conclusion $(\mathrm{A}))$.

It remains to show that Case 2 yields a contradiction.

Define $\Gamma, \tilde{\Gamma}: X \times Y \longrightarrow 2^{X \times Y}$ by

$$
\Gamma(x, y):=(X \times Y) \backslash(G(y) \times F(x)) \text { and } \tilde{\Gamma}(x, y):=(X \times Y) \backslash(\tilde{G}(y) \times \tilde{F}(x)) .
$$

Observe that

$$
\tilde{F} \text { and } \tilde{G} \text { are both surjective } \Leftrightarrow \bigcap_{(x, y) \in X \times Y} \tilde{\Gamma}(x, y)=\emptyset,
$$

which implies that hypothesis (iv) of Theorem 8 is vacuously true.

Also, $\tilde{F}$ and $\tilde{G}$ being both surjective implies that both $F$ and $G$ are surjective, which in turn amounts to saying that $\bigcap_{(x, y) \in X \times Y} \Gamma(x, y)=\emptyset$, i.e. the conclusion of the KKM Principle does not hold. This implies that $\Gamma$ is not KKM, i.e. (4) is not satisfied: there exists a convex combination $\left(x_{0}, y_{0}\right)=\sum_{i=1}^{n} \lambda_{i}\left(x_{i}, y_{i}\right)$ with

$$
\begin{aligned}
\left(x_{0}, y_{0}\right) \notin \bigcup_{i=1}^{n} \Gamma\left(x_{i}, y_{i}\right) \Leftrightarrow\left(x_{0}, y_{0}\right) & \in \bigcap_{i=1}^{n}\left(G\left(y_{i}\right) \times F\left(x_{i}\right)\right) \\
& \Leftrightarrow\left(y_{i}, x_{i}\right) \in G^{-1}\left(x_{0}\right) \times F^{-1}\left(y_{0}\right) \quad \forall i \in\{1, \ldots, n\} .
\end{aligned}
$$


Since $G^{-1}\left(x_{0}\right) \times F^{-1}\left(y_{0}\right)$ is a convex subset of $Y \times X$, it follows that $\left(y_{0}, x_{0}\right) \in$ $G^{-1}\left(x_{0}\right) \times F^{-1}\left(y_{0}\right)$, that is

$$
g\left(x_{0}, y_{0}\right)<\lambda<f\left(x_{0}, y_{0}\right) .
$$

This contradicts $g\left(x_{0}, y_{0}\right) \geq f\left(x_{0}, y_{0}\right)$ and ends the proof.

Step 2. We show now that if the nonlinear alternative holds with $X, Y$ both convex compact, then it holds with $X, Y$ both convex but only $Y$ compact. Assume that alternative (B) fails, i.e.

$$
\forall y \in Y \exists x \in X \text { with } \tilde{f}(x, y)>\lambda,
$$

which amounts to saying that the collection of open subsets $\{\{y \in Y: \tilde{f}(x, y)>\lambda\}: x \in X\}$ forms an open cover of $Y$. Since $Y$ is compact, it can be covered by a finite subcollection $\left\{\left\{y \in Y: \tilde{f}\left(x_{i}, y\right)>\lambda\right\}: x_{1}, \ldots, x_{n} \in X\right\}$. The convex hull $C:=\operatorname{Conv}\left\{x_{1}, \ldots, x_{n}\right\}$ is a convex compact subset of $X$. Theorem 3 applied to the restrictions $f, \tilde{f}, g, \tilde{g}$ to $C \times Y$ yields the nonlinear alternative:

$$
\text { A) }{ }_{C} \text { there exists } \bar{x} \in C \text { such that } \tilde{g}(\bar{x}, y) \geq \lambda \text { for all } y \in Y
$$

or

$$
\text { B) }{ }_{C} \text { there exists } \bar{y} \in Y \text { such that } \tilde{f}(x, \bar{y}) \leq \lambda \text { for all } x \in C .
$$

Obviously, if A $)_{C}$ holds, then alternative A) in Theorem 3 holds and we are done. It remains to show that $\mathrm{B})_{C}$ fails. Indeed, $\left.\mathrm{B}\right)_{C}$ implies that $\tilde{f}\left(x_{i}, \bar{y}\right) \leq \lambda$ for all $i=1, \ldots, n$; this contradicts the fact that $Y=\bigcup_{i=1}^{n}\left\{y \in Y: \tilde{f}\left(x_{i}, y\right)>\lambda\right\}$ and ends the proof.

REMARK 15. The proofs of the first two implications above are new. The proofs of the last three are essentially due to Dugundji and Granas (see $[9,10,13]$ ).

\section{References}

[1] H. Ben-El-Mechaiekh, P. Deguire, A. Granas, Points fixes et coïncidences pour les fonctions multivoques II (applications de type $\varphi$ et $\varphi^{*}$ ), C. R. Acad. Sci. Paris Sér. I Math. 295 (1982), 381-384.

[2] H. Ben-El-Mechaiekh, R. Dimand, Von Neumann, Ville, and the Minimax Theorem, STOREP Conference on the History of Decision Theory, Siena, June 2005.

[3] C. Berge, Sur une convexité régulière et ses applications à la théorie des jeux, Bull. Soc. Math. France 82 (1954), 301-315.

[4] C. Berge, Sur une propriété combinatoire des ensembles convexes, C. R. Acad. Sci. Paris 248 (1959), 2698-2699.

[5] É. Borel, La théorie du jeux et les équations intégrales à noyau symétrique gauche, C. R. Acad. Sci. Paris 173 (1921), 1304-1308; translated by L. J. Savage as The theory of play and integral equations with skew-symmetric kernels, Econometrica 21 (1953), 97-100.

[6] É. Borel, Sur les jeux où interviennent le hasard et l'habileté des joueurs, in [8], 204-221; translated by L. J. Savage as On games that involve chance and the skill of players, Econometrica 21 (1953), 101-115. 
[7] É. Borel, Sur les systèmes de formes linéaires à déterminant symétrique gauche et la théorie générale du jeu, C. R. Acad. Sci. Paris 184 (1927), 52-54; translated by L. J. Savage as On systems of linear forms of skew symmetric determinant and the general theory of play, Econometrica 21 (1953), 116-117.

[8] É. Borel, Eléments de la théorie des probabilités, 3ième édition revue et augmentée, Hermann, Paris 1924.

[9] J. Dugundji, A. Granas, KKM maps and variational inequalities, Ann. Scuola Norm. Sup. Pisa Cl. Sci. (4) 5 (1978), 679-682.

[10] J. Dugundji, A. Granas, Fixed Point Theory, Vol. I, Monografie Matematyczne 61, PWN, Warszawa 1982.

[11] K. Fan, Minimax theorems, Proc. Nat. Acad. Sci. U.S.A. 39 (1953), 42-47.

[12] M. Fréchet, Commentary on the three notes of Emile Borel, Econometrica 21 (1953), $118-124$.

[13] A. Granas, KKM maps and their applications to nonlinear problems, in: The Scottish Book, ed. R. D. Maudlin, Birkhäuser, Boston 1981.

[14] J. Kindler, A simple proof of Sion's Minimax Theorem, Amer. Math. Monthly 112 (2005), 356-358.

[15] V. L. Klee, Jr., On certain interesection properties of convex sets, Canadian J. Math. 3 (1951), 272-275.

[16] B. Knaster, C. Kuratowski, S. Mazurkiewicz, Ein Beweis des Fixpunktsatzes für n-dimensionale Simplexe, Fund. Math. 14 (1929), 132-138.

[17] H. Kneser, Sur un théorème fondamental de la théorie des jeux, C. R. Acad. Sci. Paris 234 (1952), 2418-2420.

[18] J. von Neumann, Sur la théorie des jeux, C. R. Acad. Sci. Paris 186 (1928), 1689-1691.

[19] J. von Neumann, Zur Theorie der Gesellschaftsspiele, Math. Annalen 100 (1928), 295-320; translated by S. Bargmann as On the theory of games of strategy, in: Contribution to the Theory of Games IV, eds. R. D. Luce, A. W. Tucker, supplement to Annals of Mathematics Studies No. 40, Princeton University Press, Princeton, 1959, 13-42.

[20] J. von Neumann, Über ein Ökonomisches Gleichungssystem und eine Verallgemeinerung des Brouwerschen Fixpunktsatzes, in: Ergebnisse eines Mathematischen Kolloquiums, ed. K. Menger, Vienna; translated by G. Morton as A model of general economic equilibrium, Review of Economic Studies 13 (1945), 1-9.

[21] J. von Neumann, Communication on the Borel notes, Econometrica 21 (1953), 124-127.

[22] J. von Neumann, O. Morgenstern, Theory of Games and Economic Behavior, Princeton University Press, Princeton 1944.

[23] H. Nikaidô, On von Neumann's Minimax Theorem, Pacific J. Math. 4 (1954), 65-72.

[24] M. Shiffman, On the equality $\min \max =\max \min$, and the theory of games, RAND Report RM-243, 1949.

[25] M. Sion, On general minimax theorems, Pacific J. Math. 8 (1958), 171-176.

[26] J. Ville, Sur la théorie générale des jeux où intervient l'habileté des joueurs, in: Traité du calcul des probabilités et de ses applications, Tome IV, Fasc. 2: Applications des jeux de hasard, E. Borel and Collaborators, Gauthier-Villars, Paris 1938, 105-113.

[27] H. Weyl, Elementary proof of a minimax theorem due to von Neumann, in: Contributions to the Theory of Games I, eds. H. W. Kuhn, A. W. Tucker, Annals of Mathematical Studies, no. 24, Princeton University Press, Princeton 1950, 19-25. 